\title{
Effects of electric-acoustic and acoustic-electric conversions of transducers on acoustic logging signal
}

\author{
FA Lin ${ }^{1 *}$, XIE WenYan ${ }^{2}$, TIAN Yong ${ }^{1}$, ZHAO MeiShan ${ }^{3}$, MA Li $^{4} \&$ DONG DaQun ${ }^{5}$ \\ ${ }^{1}$ School of Electronics Engineering, Xi'an University of Post and Telecommunications, Xi'an 710121, China; \\ ${ }^{2}$ PetroChina Liaohe Oilfield Company, Panjin 124013, China; \\ ${ }^{3}$ The James Franck Institute and Department of Chemistry, The University of Chicago, Chicago, IL 60637, USA; \\ ${ }^{4}$ Department of Digital Media and Art, Xi'an University of Post and Telecommunications, Xi'an 710121, China; \\ ${ }^{5}$ School of Marine Engineering, Northwest Polytechnical University, Xi'an 710071, China
}

Received October 23, 2011; accepted December 15, 2011

\begin{abstract}
Based on an acoustic logging transmission network and the engineering pattern of a sliding wave in acoustic logging, analysis and calculations have been performed in a study of the effects of the electric-acoustic and acoustic-electric conversions of the transducers on the acoustic logging signal. The results show that acoustic-electric conversion through the transducer can cause not only a serious disturbance in the signal amplitude, but also an apparent transmission delay. For engineering applications, the amplitude variation and transmission delay must be accounted for in a practical analysis of the acoustic logging signal in rocks. The results also show that with enhanced understanding and proper justification, the error caused by the acoustic-electric conversion can be significantly reduced in evaluation of the cement bond quality of a cased well, and the accuracy of rock porosity calculated using the measured acoustic velocity can be increased.
\end{abstract}

transducer, acoustic-electric conversion, transmission delay

Citation: Fa L, Xie W Y, Tian Y, et al. Effects of electric-acoustic and acoustic-electric conversions of transducers on acoustic logging signal. Chin Sci Bull, 2012, 57: 1246-1260, doi: 10.1007/s11434-012-5034-1

The amplitude information of an acoustic signal and its propagation speed in rocks has been widely used in the petroleum industry. For example, in acoustic logging, the applications and developments include: (1) use of the amplitudes of the head wave and the later arrivals of the acoustic logging signal to evaluate the cement bond quality of cased wells; (2) use of the $P$-wave velocity of the acoustic signal to calculate rock porosity by the Wyllie formula [1] and to calibrate seismic data; (3) use of the $P$-and $S$-wave velocities to calculate the mechanical parameters of rock (such as Young's modulus and the Poisson ratio); (4) use of the acoustic logging data to construct the atoms in a seismic wavelet dictionary. In seismic exploration, the applications and developments include: (1) use of the $P$-wave amplitude information to perform amplitude variation with offset

*Corresponding author (email: fa_yy@yahoo.com.cn)
(AVO) analysis to search for oil reservoirs; and (2) use of the $P$-wave speed of a seismic wavelet to perform timedepth conversion for inversion of the rock structure in the Earth's interior. Accurate measurements of both the amplitude and the propagation speed of the acoustic signal in rocks are therefore very important for correct inversions and interpretations of acoustic logging and seismic data. In the research conducted by many geophysicists to accurately obtain the amplitude, propagation speed, phase and frequency spectrum of an acoustic signal propagating in rock, considerable attention has been paid to the effects of the rock properties on the measured acoustic signals. Cong et al. [2] performed experimental studies on the effects of the rock porosity on acoustic resonance spectroscopy. Fa et al. $[3,4]$ calculated the effects of rock anisotropy on the propagation speed and reflection amplitude of a seismic wavelet. The purpose of these studies is to obtain accurate infor- 
mation on the amplitude and propagation speed of the acoustic signal (acoustic logging signal or seismic reflection wavelet) for correct inversions of the acoustic logging and seismic exploration data. However, they barely discussed the issue about the effects of the acoustic-electric conversion of transducers (piezoelectric transducer or geophone) on the acoustic signal (acoustic logging signal or seismic reflection wavelet).

In this paper, on the basis of published work by various researchers [4-10], we use an acoustic logging transmission network model $[11,12]$ to perform calculations and discuss the effects of the acoustic-electric conversion of the transducer on the acoustic logging signals.

Theoretically, if the propagation medium is an ideal elastic body or its acoustic attenuation coefficient is very small, then applying two receivers at different ranges to measure the arrival time-difference of the acoustic signal can eliminate the effects of acoustic-electric conversion at the transducer on the propagation speed of the acoustic signal. However, for rocks with stronger acoustic attenuation properties (especially shale), the effects of the transducer on acoustic-electric conversion can cause the waveform of the acoustic signal wavelet to create greater variations in its propagation process. The acoustic attenuation coefficient of a rock and the propagation speed of the acoustic wave depend on the frequency of the acoustic wave. The acoustic signal wavelet radiated by a source usually consists of multiple frequency components. For each frequency component, as the acoustic signal propagates in the rock, there is a unique attenuation with a given speed. The acoustic signal arriving at the receiver therefore creates not only a propagation delay but also waveform variations.

Generally, in a practical engineering network, the distance difference between two receivers in an acoustic logging tool is about $0.4 \mathrm{~m}$ and the distance from the source to the nearest receiver is $0.8-1.4 \mathrm{~m}$. For a rock formation with stronger acoustic attenuation properties, the waveforms of the acoustic logging signals arriving at the two receiver positions can be quite different. The major reasons for this difference are: (i) the acoustic logging signal consists of sliding $P$ - and $S$-waves coming from the borehole wall, as well as first- and multi-order reflection waves in the borehole fluid; (ii) when the sliding $P$-wave and/or sliding $S$-wave propagates to the two receiver positions, the different propagation distances cause each frequency component of the sliding waves to create different attenuation values; (iii) each frequency component of these waves has a different propagation velocity in the rock formation around the borehole and in the fluid in the borehole. Therefore, for the same propagation distance, the arrival time of each frequency component traveling from the source to the receiver is different; and (iv) because of the different source-receiver distances for the two receivers, the propagation paths and distances for the first-order reflection wave or the multiorder reflection wave in the borehole fluid are different; the different propagation distances can cause each frequency component of the above reflection waves to create different attenuation values. While the different propagation paths make the corresponding first-order reflection wave and/or multi-order reflection waves have different incident angles from the borehole fluid to the borehole wall, they also have different reflection coefficient values.

In this paper, we emphasize the effects of the acousticelectric conversion of a receiver on the head wave amplitude and the propagation speed of the measured acoustic logging signal. We use two different Tsang wavelets [6] with the same center frequency $(10 \mathrm{kHz})$. Different damping coefficients are used so that they effectively create the waveform difference of the acoustic logging signals arriving at the two receivers. When these two Tsang wavelets are injected into the receiver, the acoustic-electric conversion of the receiver would cause the acoustic logging signal to create an amplitude variation and an apparent transmission delay. It is also noted that to evaluate the cement bond quality of a cased well and to obtain the porosity of the rock formation accurately using acoustic logging data (or data measured from rock samples), both the amplitude variation and the transmission delay of the acoustic signal caused by the acoustic-electric conversion should be considered. In the acoustic logging transmission network model, the acoustic logging signal arriving at the receiver transducer position is an acoustic pressure signal, while the measured acoustic logging signal is an electric signal converted by the receiver from the above acoustic signal. Both of these signals have apparent differences in waveform, amplitude spectrum and phase. The total transmission time of the signal wavelet is equal to the sum of the electric-acoustic conversion time of the source transducer, the travel time of the acoustic logging signal in the propagation media (borehole fluid and the formation around the borehole) and the acoustic-electric conversion time of the receiver transducer. In Section 1, based on the engineering pattern of an acoustic logging sliding wave propagating on the borehole wall, we use the acoustic logging transmission network model to analyze the amplitude variation and transmission delay of the acoustic logging signal caused by the acoustic-electric conversion of the receiver. The calculated results and discussions are presented in Section 2, and a short concluding remark is given in Section 3.

\section{Modeling}

Consider a thin spherical-shell piezoelectric transducer polarized in the radial direction, which is used as the equivalent of a point source and a point receiver [13]. A logging tool is placed in a fluid-filled cylindrical borehole with radius $a$, which is embedded in an infinitely large medium (see Figure 1). We pay particular attention to the effects of the propagation media (the borehole fluid and the formation 


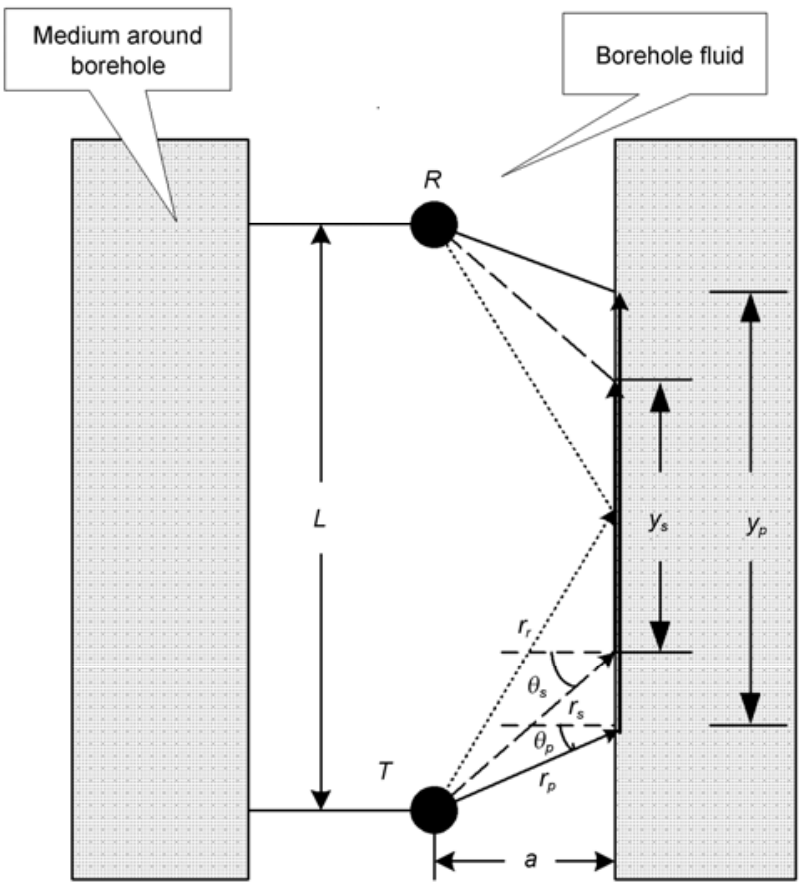

Figure 1 A schematic diagram of the geometrical configuration of acoustic logging, where the solid line is the propagation path of the sliding $P$-wave, the dashed line is that of the sliding $S$-wave, and the point line is that of the first-order reflection wave.

around the borehole) on the sliding $P$-wave, the sliding $S$-wave, and the first-order reflection wave. As shown in Figure 1, the source-transducer $(T)$ is located on the borehole axis and the distance from the receiver transducer $(R)$ on the borehole axis to the source transducer is $L$. The incident critical angles of the $P$-wave and the $S$-wave from the borehole fluid to the formation are denoted by $\theta_{p}$ and $\theta_{s}$ respectively.

We also consider the acoustic source as an electricacoustic filter, the propagation media as acoustic filters, and the receiver as an acoustic-electric filter. Based on these considerations, the geometrical configuration of acoustic logging (Figure 1) may be described as an acoustic logging transmission network (see Figure 2).

Within the acoustic logging transmission network model, the driving voltage signal $u_{1}(t)$ is defined as the input signal of the system. $h_{1}(t)$ is the electric-acoustic impulse response of the source transducer $(T)$, while $h_{3}(t)$ is the acoustic-

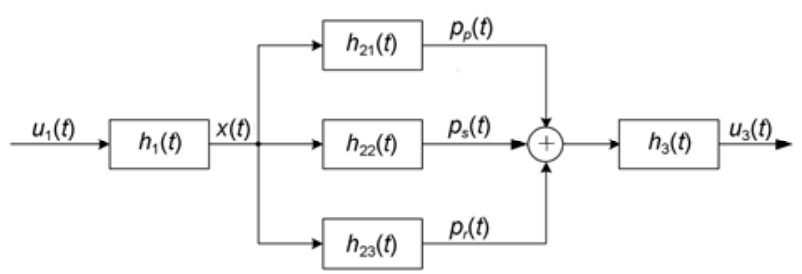

Figure 2 An acoustic logging transmission network for the acoustic logging engineering pattern of sliding waves. electric impulse response of the receiver transducer $(R)$. When $T$ is excited by $u_{1}(t), T$ radiates an acoustic pressure signal wavelet $x(t)$ outwards. The acoustic impulse responses from the propagation media are denoted for the sliding $P$-wave, the $S$-wave, and the first-order reflection wave, as $h_{21}(t), h_{22}(t)$ and $h_{23}(t)$, respectively. As shown in Figure 1, the acoustic logging signal can be obtained from various traveling waves from the source transducer to the receiver transducer, i.e. $p_{p}(t)$ for the acoustic pressure of the sliding $P$-wave (solid line), $p_{s}(t)$ for that of the sliding $S$-wave (dashed line), and $p_{r}(t)$ for that of the first-order reflection wave (point-line). In Figure 2, the notation sign " $\oplus$ " is used for an adder.

As shown in Figure 2, when $u_{1}(t)$ is injected into $T$, the acoustic logging transmission network produces a final electric output signal $u_{3}(t)$, which can be written as

$$
u_{3}(t)=u_{1}(t) * h_{1}(t) *\left[\sum_{i=1}^{3} h_{2 i}(t)\right] * h_{3}(t) .
$$

This equation represents the critical relationship between the driving voltage signal, the propagation media, and the measured acoustic logging signal. In the following sections, we analyze in detail the various transmission processes for the acoustic logging signal within the acoustic logging transmission network.

\subsection{Transducer}

Thin spherical-shell transducers with average radius $r_{b}$ and wall thickness $l_{t}$, polarized in the radial direction are taken to be the source and the receiver. Figure 3 shows two equivalent circuits for the source and the receiver, e.g. Figure 3(a) for the source transducer and Figure 3(b) for the receiver transducer. The various symbol notations used in Figure 3 are defined in Table 1.
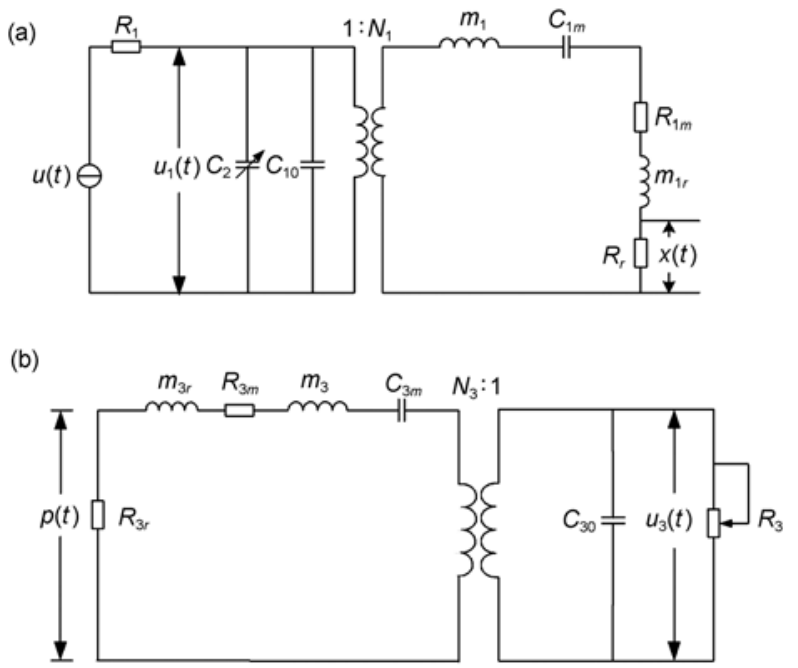

Figure 3 The equivalent circuits of the transducers. (a) The source transducer; (b) the receiver transducer. 
Table 1 Definitions of the symbols shown in Figure 3

\begin{tabular}{|c|c|c|}
\hline Symbol & Description & Expression \\
\hline$R_{1}$ & Output resistance of driving circuit & \\
\hline$u(t)$ & Driving voltage signal & \\
\hline$u_{1}(t)$ & $\begin{array}{l}\text { Voltage signal of the electric } \\
\text { terminals of } T\end{array}$ & \\
\hline$x(t)$ & $\begin{array}{l}\text { Acoustic pressure signal radiated } \\
\text { by } T\end{array}$ & \\
\hline$p(t)$ & $\begin{array}{l}\text { Acoustic pressure signal arriving } \\
\text { at position where } R \text { is located }\end{array}$ & \\
\hline$u_{3}(t)$ & Electrical output signal of $R$ & \\
\hline$R_{3}$ & Load resistance of $R$ & \\
\hline$C_{2}$ & Parallel capacity & \\
\hline$C_{i 0}$ & Clamped capacitance of transducer & $C_{i 0}=4 \pi r_{b}^{2} \varepsilon_{33}\left(1-k_{13}^{2}\right) / l_{t}$ \\
\hline$N_{i}$ & $\begin{array}{l}\text { Mechanical-electrical conversion } \\
\text { coefficient of transducer }\end{array}$ & $N_{i}=4 \pi r_{b} d_{31} / S_{c}$ \\
\hline$m_{i}$ & Mass of transducer & $m_{i}=4 \pi r_{b}^{2} l_{t} \rho_{p}$ \\
\hline$C_{i m}$ & $\begin{array}{l}\text { Elastic stiffness of transducer } \\
\text { material }\end{array}$ & $C_{i m}=\left(s_{11}+s_{12}\right) / 8 \pi l_{t}$ \\
\hline$m_{i r}$ & Radiation mass of transducer & $m_{i r}=4 \pi \rho_{m} r_{b}^{3} /\left(1+k_{m}^{2} r_{b}^{2}\right)$ \\
\hline$R_{i r}$ & Radiation resistance of transducer & $R_{i r}=4 \pi k_{m}^{2} \rho_{m} v_{m} r_{b}^{4} /\left(1+k_{m}^{2} r_{b}^{2}\right)$ \\
\hline$R_{i m}$ & Friction force resistance & $R_{i m}=0.8 \pi r_{b}^{2} \rho_{m} v_{m}$ \\
\hline$Z_{m}$ & $\begin{array}{l}\text { Acoustic impedance of coupling } \\
\text { medium around transducer }\end{array}$ & \\
\hline
\end{tabular}

(i) Source transducer. As shown in Figure 3(a) for the source transducer, a Laplace transform of the electricacoustic transmission function can be written as

$$
H_{1}(s)=\frac{s d_{1}}{s^{3}+a_{1} s^{2}+b_{1} s+c_{1}}
$$

where $a_{1}=b_{11} / a_{11}, \quad b_{1}=c_{11} / a_{11}, \quad c_{1}=d_{11} / a_{11}, \quad d_{1}=e_{11} / a_{11}, \quad e_{11}=$ $N R_{r} C_{1 m}, d_{11}=1, a_{11}=R_{1} C_{10} C_{1 m}\left(m_{1}+m_{2}\right), b_{11}=R_{1} C_{10} C_{1 m}\left(R_{m}+R_{r}\right)$ $+C_{1 m}\left(m_{1}+m_{r}\right)$ and $c_{11}=N^{2} R_{1} C_{1 m}+R_{1} C_{10}+C_{1 m}\left(R_{m}+R_{r}\right)$.

The discriminant of the third-order equation $\left(s^{3}+s^{2} a_{1}+\right.$ $s b_{1}+c_{1}=0$ ) in eq. (2) is given as

$$
\Delta_{1}=\left(\frac{q_{1}}{2}\right)^{2}+\left(\frac{p_{1}}{2}\right)^{3}
$$

where $\quad x_{1}=\sqrt[3]{-\frac{q_{1}}{2}+\sqrt{\Delta_{1}}}, \quad y_{1}=\sqrt[3]{-\frac{q_{1}}{2}-\sqrt{\Delta_{1}}}, \quad q_{1}=c_{1}+$ $\frac{2 a_{1}^{3}}{27}-\frac{a_{1} b_{1}}{3}$ and $p_{1}=b_{1}-\frac{a_{1}^{2}}{3}$.

Invoking the relationship between Laplace and Fourier transforms, its Fourier transform may be written as

$$
H_{1}(\omega)=\frac{j \omega d_{1}}{-j \omega^{3}-\omega^{2} a_{1}+j \omega b_{1}+c_{1}} .
$$

(1) In case where $\Delta_{1}<0$, the third-order equation $\left(s^{3}+\right.$ $\left.s^{2} a_{1}+s b_{1}+c_{1}=0\right)$ has three independent real roots $\left(s_{1}, s_{2}, s_{3}\right)$. The source transducer is in an over-damped mode. The re- sponse to an electric-acoustic impulse can be written as

$h_{1}(t)=h_{11}(t)+h_{12}(t)+h_{13}(t)=K_{10} \mathrm{e}^{s_{1} t}+K_{11} \mathrm{e}^{s_{2} t}+K_{12} \mathrm{e}^{s_{3} t}$,

where $s_{1}=\frac{a_{1}}{3}-\left(x_{1}+y_{1}\right), s_{2}=\frac{a_{1}}{3}+\frac{x_{1}+y_{1}}{2}, s_{3}=\frac{x_{1}+y_{1}}{2}-\frac{5 a_{1}}{3}$,

$K_{10}=\frac{4 a_{1} d_{1}-12\left(x_{1}+y_{1}\right) d_{1}}{27\left(x_{1}+y_{1}\right)^{2}-36 a_{1}\left(x_{1}+y_{1}\right)}, K_{11}=\frac{2 a_{1}+3\left(x_{1}+y_{1}\right) d_{1}}{18 a_{1}\left(x_{1}+y_{1}\right)}$

and $K_{12}=\frac{3\left(x_{1}+y_{1}\right)-10 a_{1}}{24 a_{1}^{2}-18 a_{1}\left(x_{1}+y_{1}\right)} d_{1}$.

(2) When $\Delta_{1}=0$, the third-order equation $\left(s^{3}+s^{2} a_{1}+s b_{1}+\right.$ $c_{1}=0$ ) has three real roots and two of them are the same, i.e. $s_{1}=s_{2} \neq s_{3}$. The source transducer is in a critically-damped mode. The response to the electric-acoustic impulse is given as

$$
h_{1}(t)=K_{20} \mathrm{e}^{s_{1} t}+\left(K_{21} t+K_{22}\right) \mathrm{e}^{s_{2} t},
$$

where $s_{1}=\frac{a_{1}}{3}-\left(x_{1}+y_{1}\right), s_{2}=s_{3}=\frac{a_{1}}{3}+\frac{x_{1}+y_{1}}{2}, \quad K_{20}=$ $\frac{2 a_{1}+3\left(x_{1}+y_{1}\right)}{9\left(x_{1}+y_{1}\right)} d_{1}, \quad K_{21}=\frac{12\left(x_{1}+y_{1}\right)-4 a_{1}}{27\left(x_{1}+y_{1}\right)^{2}}$ and $K_{22}=$ $\frac{2 a_{1}+3\left(x_{1}+y_{1}\right)}{9\left(x_{1}+y_{1}\right)}$.

(3) In the case of $\Delta_{1}>0$, the third-order equation $\left(s^{3}+\right.$ $\left.s^{2} a_{1}+s b_{1}+c_{1}=0\right)$ has one real root $\left(s_{1}=-\alpha_{1}\right)$ and two complex conjugate roots, $s_{2,3}=\beta_{1} \pm j \omega_{1}$. The source transducer is in an oscillation mode. The response to an electric-acoustic impulse is given by

$$
h_{1}(t)=K_{30} \mathrm{e}^{-\alpha_{1} t}+2\left|K_{31}\right| \mathrm{e}^{-\beta_{1} t} \cos \left(\omega_{1} t-\theta_{1}\right),
$$

with a center (or) resonance frequency

$$
f_{1}=\frac{\omega_{1}}{2 \pi}=\frac{\sqrt{3}}{4 \pi}\left(x_{1}-y_{1}\right)
$$

where $\alpha_{1}=\frac{a_{1}}{3}-\left(x_{1}+y_{1}\right), \beta_{1}=\frac{a_{1}}{3}+\frac{x_{1}+y_{1}}{2}, \omega_{1}=\frac{\sqrt{3}\left(x_{1}-y_{1}\right)}{2}$, $\theta_{1}=\arctan \left(\frac{\alpha_{1} \beta_{1}-\beta_{1}^{2}-\omega_{1}^{2}}{\omega_{1} \beta_{1}}\right), \quad K_{30}=U_{0} d_{1} /\left(-\beta_{1}+\alpha_{1}-j \omega_{1}\right)\left(-\beta_{1}+\right.$ $\left.\alpha_{1}+j \omega_{1}\right)$ and $K_{31}=\frac{U_{0} d_{1} \sqrt{\omega_{1}^{2}+\left(\beta_{1}-\alpha_{1}\right)^{2}}}{2 \omega_{1}^{3}+2 \omega_{1}\left(\beta_{1}-\alpha_{1}\right)^{2}}$.

(ii) Receiver transducer. The Laplace transform of the acoustic-electric transmission function for the receiver transducer, as shown in Figure 3(b), can be written as

$$
H_{3}(s)=\frac{s d_{3}}{s^{3}+s^{2} a_{3}+s b_{3}+c_{3}} .
$$

Its corresponding acoustic-electric Fourier transform is 
given by

$$
H_{3}(\omega)=\frac{j \omega d_{3}}{-j \omega^{3}-\omega^{2} a_{3}+j \omega b_{3}+c_{3}}
$$

where $a_{3}=b_{33} / a_{33}, \quad b_{3}=c_{33} / a_{33}, \quad c_{3}=d_{33} / a_{33}, d_{3}=e_{33} / a_{33}, \quad a_{33}=$ $R_{3} C_{30} C_{3 m} m_{3}+R_{3} C_{30} C_{3 m} m_{r 3}, b_{33}=R_{3} C_{30} C_{3 m} R_{r 3}+m_{3} C_{3 m}+m_{r 3} C_{3 m}+$ $R_{3} C_{30} C_{3 m} R_{m 3}, c_{33}=R_{3} C_{30}+C_{3 m} R_{m 3}+N^{2} R_{3} C_{3 m}+C_{3 m} R_{r 3}, d_{33}=1$ and $e_{33}=N R_{3} C_{3 m}$.

The discriminant of the third-order equation $\left(s^{3}+s^{2} a_{3}+\right.$ $s b_{3}+c_{3}=0$ ) in eq. (9) is shown as

$$
\Delta_{3}=\left(\frac{p_{3}}{3}\right)^{3}+\left(\frac{q_{3}}{2}\right)^{2}
$$

where $x_{3}=\sqrt[3]{-\frac{q_{3}}{2}+\sqrt{\Delta_{3}}}, y_{3}=\sqrt[3]{-\frac{q_{3}}{2}-\sqrt{\Delta_{3}}}, q_{3}=c_{3}+\frac{2 a_{3}^{3}}{27}-$ $\frac{a_{3} b_{3}}{3}$, and $p_{3}=b_{3}-\frac{a_{3}^{2}}{3}$.

(1) In the case of $\Delta_{3}<0$, the third-order equation $\left(s^{3}+s^{2} a_{3}+\right.$ $\left.s b_{3}+c_{3}=0\right)$ has three different real roots $\left(s_{1}, s_{2}, s_{3}\right)$ and the receiver transducer is in an over-damped mode. The response to the acoustic-electric impulse is then

$$
h_{3}(t)=\tilde{K}_{10} \mathrm{e}^{s_{1} t}+\tilde{K}_{11} \mathrm{e}^{s_{2} t}+\tilde{K}_{12} \mathrm{e}^{s_{3} t},
$$

where $s_{1}=\frac{a_{3}}{3}-\left(x_{3}+y_{3}\right), s_{2}=\frac{a_{3}}{3}+\frac{x_{3}+y_{3}}{2}, s_{3}=\frac{x_{3}+y_{3}}{2}-$

$\frac{5 a_{3}}{3}, \tilde{K}_{11}=\frac{2 a_{3}+3\left(x_{3}+y_{3}\right)}{18 a_{3}\left(x_{3}+y_{3}\right)} d_{3}, \tilde{K}_{10}=\left(4 a_{3} d_{3}-12\left(x_{3}+y_{3}\right) d_{3}\right) /$

$\left(27\left(x_{3}+y_{3}\right)^{2}-36 a_{3}\left(x_{3}+y_{3}\right)\right)$ and $\tilde{K}_{12}=\left(3\left(x_{3}+y_{3}\right)-10 a_{3}\right) d_{3} /$ $\left(24 a_{3}^{2}-18 a_{3}\left(x_{3}+y_{3}\right)\right)$.

(2) In the case of $\Delta_{3}=0$, the third-order equation $\left(s^{3}+\right.$ $\left.s^{2} a_{3}+s b_{3}+c_{3}=0\right)$ has three real roots and two of them are identical (i.e. $s_{1}=s_{2} \neq s_{3}$ ), and the receiver transducer is in a critically damped mode. The response to the acousticelectric impulse is then given as

$$
h_{3}(t)=\tilde{K}_{20} \mathrm{e}^{s_{1} t}+\left(\tilde{K}_{21} t+\tilde{K}_{22}\right) \mathrm{e}^{s_{2} t},
$$

where $s_{1}=\frac{a_{3}}{3}-\left(x_{3}+y_{3}\right), \quad s_{s}=s_{3}=\frac{a_{3}}{3}+\frac{x_{3}+y_{3}}{2}, \quad \tilde{K}_{20}=$ $\frac{2 a_{3}+3\left(x_{3}+y_{3}\right)}{9\left(x_{3}+y_{3}\right)} d_{3}, \quad \tilde{K}_{21}=\frac{12\left(x_{3}+y_{3}\right)-4 a_{3}}{27\left(x_{3}+y_{3}\right)^{2}}$ and $\tilde{K}_{22}=$ $\frac{2 a_{3}+3\left(x_{3}+y_{3}\right)}{9\left(x_{3}+y_{3}\right)}$.

(3) In the case of $\Delta_{3}>0$, the third-order equation $\left(s^{3}+s^{2} a_{3}+\right.$ $s b_{3}+c_{3}=0$ ) has one real root $s_{1}$ and two complex conjugate roots $s_{3}=s_{2}^{*}$. In this case, the receiver transducer is in an oscillation mode. Its response to the acoustic-electric impulse yields

$$
h_{3}(t)=\tilde{K}_{30} \mathrm{e}^{-\alpha_{3} t}+\tilde{K}_{31} \mathrm{e}^{-\beta_{3} t} \cos \left(\omega_{3} t+\theta_{3}\right)
$$

with a center (or resonance) frequency

$$
f_{3}=\frac{\omega_{3}}{2 \pi}=\frac{\sqrt{3}}{4 \pi}\left(x_{3}-y_{3}\right),
$$

where $s_{1}=-\alpha_{3}, s_{2,3}=-\beta_{3} \pm j \omega_{3}, A_{3}=\frac{x_{3}+y_{3}}{2}, B_{3}=\frac{x_{3}-y_{3}}{2}$, $\omega_{3}=\sqrt{3} B_{3}, \quad \alpha_{3}=\frac{a_{3}}{3}-2 A_{3}, \quad \beta_{3}=\frac{a_{3}}{3}+A_{3}, \quad \tilde{K}_{30}=d_{3} \alpha_{3} /$ $\left(\left(-\alpha_{3}+\beta_{3}\right)^{2}+\omega_{3}^{2}\right), \quad \tilde{K}_{31}=\frac{d_{3} E_{3}}{\alpha_{3}^{2}-\beta_{3}^{2}+\omega_{3}^{2}} \quad$ and $\quad \theta_{3}=\operatorname{arctg}$ $\left(\frac{\beta_{3}^{2}-\omega_{3}^{2}}{\omega_{3} \alpha_{3}}\right)$.

It is noted that in both the critically damped mode and the over-damped mode of the transducer, it radiates a pressure pulse or produces a forced vibration. The receiver transducer can create only an electric pulse signal or an electric signal wavelet from the acoustic signal wavelet.

For both the over-damped mode and the critically damped mode, the transducer has low efficiencies for the electric-acoustic and acoustic-electric conversions. In an oscillation mode, the source transducer radiates an acoustic signal wavelet and the receiver transducer converts the acoustic signal wavelet into an electric signal wavelet. When the center frequency of the driving voltage signal is coincident with or very near the center frequency of the source transducer, the source yields the highest electric-acoustic conversion efficiency. Similarly, when the center frequency of the received acoustic logging signal is the same as or near to the center frequency of the receiver transducer, the receiver yields the highest acoustic-electric conversion efficiency. When both the source transducer and the receiver transducer are in the critically damped mode, their transient processes will be at their shortest.

\subsection{Propagation media}

(i) Propagation time of acoustic logging signal in propagation media. To emphasize the issue of the effect of the acoustic-electric conversion of the receiver transducer on the amplitude and transmission delay of the acoustic logging signal, we consider the following simplifications: (1) only the effects of the acoustic attenuation of the media on the magnitude of each frequency component of the acoustic logging signal are considered; (2) neglect the effect of acoustic velocity dispersion on the acoustic logging signal, i.e. assume that the propagation speeds of all frequency components of the acoustic logging signal are identical; (3) consider the borehole fluid and the formation around the borehole to be virtually isotropic; and (4) approximate the energy velocity by the phase velocity for the acoustic logging signal. Based on these simplifications, in the incident 
critical angle directions of the sliding $P$ - and $S$-waves (see Figure 1), the travel time of the acoustic signal from the source transducer to the wall of the borehole can be calculated from $t_{f p}=r_{p} / v_{f}$ and $t_{f s}=r_{s} / v_{f}$, respectively. Here, $v_{f}$ is the propagation speed of the acoustic signal in the borehole fluid. Similarly, the propagation times in the formation for the sliding $P$-wave and the sliding $S$-wave are $t_{p 0}=y_{p} / v_{p}$ and $t_{s 0}=y_{s} / v_{s}$, where $v_{p}$ and $v_{s}$ are the propagation speeds of the sliding $P$-wave and the sliding $S$-wave in the formation, respectively. Therefore, the total propagation time of the sliding $P$-wave in the media is

$$
t_{p}=t_{p 0}+2 t_{f p} .
$$

The propagation time for the sliding $S$-wave in the media is given by

$$
t_{s}=t_{s 0}+2 t_{f s} .
$$

The propagation time for the first-order reflection wave in the borehole fluid is written as

$$
t_{r}=\frac{2 r_{r}}{v_{f}}
$$

The various symbol definitions related to eqs. (16)-(18) are $r_{p}=\frac{a}{\cos \theta_{p}}, r_{s}=\frac{a}{\cos \theta_{s}}, r_{r}=\sqrt{\frac{L^{2}}{4}+a^{2}}, y_{p}=L-2 a \tan \theta_{p}$, $y_{s}=L-2 a \tan \theta_{s}, \theta_{p}=\arcsin \left(\frac{v_{f}}{v_{p}}\right)$, and $\theta_{s}=\arcsin \left(\frac{v_{f}}{v_{s}}\right)$.

(ii) Acoustic attenuation. Because of the viscosity of the propagation media, acoustic attenuation will be created in the acoustic logging signal propagation process. For the monochromatic $P$-wave and $S$-wave, we start by writing the particle displacements during the propagation of the waves in the $r$-direction, such that

$$
\begin{aligned}
& u_{p}=\exp \left[-\sigma_{p} r\right] \cos \left(\omega t-k_{p} r\right) \\
& u_{s}=\exp \left[-\sigma_{s} r\right] \cos \left(\omega t-k_{s} r\right)
\end{aligned}
$$

In these equations, $\omega$ is an angular frequency; and $k_{p}$ and $k_{s}$ are the wave numbers for the monochromatic $P$-wave and $S$-wave, respectively. For the particle displacements around the borehole, the acoustic attenuation coefficients for $P$ - and $S$-waves are [14]

$$
\begin{gathered}
\sigma_{p}=\frac{\omega^{2}}{2} \frac{\eta_{11}}{c_{11}} \sqrt{\frac{\rho}{c_{11}}} \\
\sigma_{s}=\frac{\omega^{2}}{2} \frac{\eta_{44}}{c_{44}} \sqrt{\frac{\rho}{c_{44}}} .
\end{gathered}
$$

The acoustic attenuation coefficient of the borehole fluid is given by

$$
\sigma_{f}=\frac{\omega^{2}}{2} \frac{\eta_{11}^{\prime}}{c_{11}^{\prime}} \sqrt{\frac{\rho^{\prime}}{c_{11}^{\prime}}}
$$

where $\rho$ is the density of the formation, and $c_{11}^{\prime}, \eta_{11}^{\prime}$ and $\rho^{\prime}$ are the elements of the stiffness matrix, the viscosity matrix, and the density of borehole fluid, respectively.

Because the acoustic-attenuation coefficients $\left(\sigma_{p}, \sigma_{s}, \sigma_{f}\right)$ of the media are functions of the frequency, the acoustic attenuation created for each frequency component of the acoustic logging signal is different from any other, i.e. the waveform of the acoustic logging signal will create variations during the propagation process.

(iii) Scattering coefficient of borehole wall. For the benefit of the discussion, we consider the effect of the reflection coefficient from the borehole fluid to the formation around the borehole on the first-order reflection wave. In such a case, the reflection coefficient can be written as [2]

$$
R=\frac{-z+z_{p} \cos ^{2} 2 \theta_{p}+z_{s} \sin ^{2} 2 \theta_{p}}{z+z_{p} \cos ^{2} 2 \theta_{p}+z_{s} \sin ^{2} 2 \theta_{p}}
$$

with $\quad z=\frac{\rho^{\prime} v_{f}}{\cos \theta_{p}}, \quad z_{p}=\rho v_{p}, \quad z_{p}=\rho v_{p} \quad$ and $\quad \theta_{p}=$ $\arcsin \left(\frac{v_{f}}{v_{p}}\right)$.

(iv) Expressions for acoustic logging signal in every logging tache. When the source transducer is excited by the driving voltage signal, the radiated acoustic pressure signals can be expressed as

$$
x(t)=u_{1}(t) * h_{1}(t)
$$

The corresponding particle displacements can be shown in the time and frequency domains to be

$$
\begin{gathered}
x_{1}(t)=\int \frac{x(t)}{R_{r}} \mathrm{~d} t=\int \frac{u_{1}(t) * h_{1}(t)}{R_{r}} \mathrm{~d} t \\
X_{1}(j \omega)=\int_{-\infty}^{+\infty} x_{1}(t) \exp [-j \omega t] \mathrm{d} t .
\end{gathered}
$$

For the sliding $P$-wave, the $S$-wave, and the first-order reflection wave, each frequency component of the particle displacements at the receiver transducer position can be expressed as

$$
\begin{gathered}
D_{p}(j \omega)=X_{1}(j \omega) \exp \left[-2 \sigma_{f} r_{p}-\sigma_{p} y_{p}\right] \exp \left[-j \omega t_{p}\right], \\
D_{s}(j \omega)=X_{1}(j \omega) \exp \left[-2 \sigma_{f} r_{s}-\sigma_{s} y_{s}\right] \exp \left[-j \omega t_{s}\right], \\
D_{r}(j \omega)=X_{1}(j \omega) \exp \left[-2 \sigma_{f} r_{r}\right] \exp \left[-j \omega t_{r}\right] R_{p} .
\end{gathered}
$$

Each frequency component of the corresponding particle 
velocities at the receiver transducer position are given by

$$
V_{3 p}(j \omega)=j \omega X_{1}(j \omega) \exp \left[-2 \sigma_{f} r_{p}-\sigma_{p} y_{p}\right] \exp \left[-j \omega t_{p}\right],
$$

$$
\begin{gathered}
V_{3 s}(j \omega)=j \omega X_{1}(j \omega) \exp \left[-2 \sigma_{f} r_{s}-\sigma_{s} y_{s}\right] \exp \left[-j \omega t_{s}\right], \\
V_{3 r}(j \omega)=j \omega X_{1}(j \omega) \exp \left[-2 \sigma_{f} r_{r}\right] \exp \left[-j \omega t_{r}\right] R_{p} .
\end{gathered}
$$

Therefore, the acoustic pressure signal received by the receiver transducer is a summation of the contributions of the various signals of the sliding $P$-wave, $S$-wave, and first-order reflection wave:

$$
p(t)=p_{p}(t)+p_{s}(t)+p_{r}(t) .
$$

In the frequency domain, it is written as

$$
\begin{aligned}
P(j \omega)= & P_{p}(j \omega)+P_{s}(j \omega)+P_{r}(j \omega) \\
= & {\left[V_{3 p}(j \omega)+V_{3 s}(j \omega)+V_{3 r}(j \omega)\right] R_{3 r} } \\
= & j \omega X_{1}(j \omega)\left\{\exp \left[-2 \sigma_{f} r_{p}-\sigma_{p} y_{p}\right] \exp \left[-j \omega t_{p}\right]\right. \\
& +\exp \left[-2 \sigma_{f} r_{s}-\sigma_{s} y_{s}\right] \exp \left[-j \omega t_{s}\right] \\
& \left.+\exp \left[-2 \sigma_{f} r_{r}\right] \exp \left[-j \omega t_{r}\right] R_{p}\right\} R_{3 r} .
\end{aligned}
$$

Clearly, $\left[p(t), p_{p}(t), p_{s}(t), p_{r}(t)\right]$ are the inverse Fourier transforms of $\left[p(j \omega), p_{r}(j \omega), p_{p}(j \omega), p_{s}(j \omega)\right]$.

In practice, the measured acoustic logging signal is the output electric signal from the receiver transducer. It can be expressed either in the time domain or in the frequency domain as

$$
\begin{aligned}
u_{3}(t) & =p(t) * h_{3}(t), \\
U_{3}(j \omega) & =P(j \omega) H_{3}(j \omega) .
\end{aligned}
$$

\section{Results of calculations and discussion}

In the following calculations, let the acoustic source be a thin spherical-shell transducer that is composed of the piezoelectric material PZT-7A, the coupling medium around the transducer be the transformer oil, the output impedance of the driving circuit be $50 \Omega$, and the source-receiver spacing be $1.3224 \mathrm{~m}$. The parameter values and related physical data are listed in Table 2. The physical parameters of the borehole fluid and the formation around the borehole are given in Table 3.

\subsection{Electrical-acoustic conversion of the source-trans- ducer}

For the cases where the parallel capacity $C_{2}$ does not exist (i.e. $C_{2}=0$ ), as shown in Figure 3(a), and when $R_{1 m}$ is set to 1.0, 5.0 and 12.0 (in unit $\pi r_{b}^{2} Z_{m}$ ), the calculated $\Delta_{1}$ values are equal to $4.4155,2.8355$ and 2.7753 (in unit $10^{31}$ ), respectively. The source-transducer is therefore in an oscillation mode. In this case, we have calculated the normalized electric-acoustic impulse response, as shown in Figure 4. We found that the maxima of the impulse responses, in both the time and frequency domains, decrease with respect to the friction force resistance $\left(R_{1 m}\right)$. The calculated results in Figure 4 show that the heat dissipation increases with respect to the friction force resistance. When the friction force resistance is increased from $1 \pi r_{b}^{2} Z_{m}$ to $12 \pi r_{b}^{2} Z_{m}$, the center frequency of the source-transducer is decreased, but over a very small range.

When the friction force resistance is set at $0.8 \pi r_{b}^{2} Z_{m}$ and the capacitance $\left(C_{2}\right)$ is taken as $0.0,0.3$ and 0.6 (in $\left.\mu \mathrm{F}\right)$, the calculated $\Delta_{1}$ values are equal to $4.4379,1.0528$ and 5.7249 (in units of $10^{31}$ ), respectively. The source transducer is again in an oscillation mode. The normalized electricacoustic impulse responses are calculated, as shown in Figure 5. The figure shows that the maxima of the electricacoustic impulse response decrease with respect to increasing capacitance. The resonance frequency $\left(f_{1}\right)$ increases only slightly when the capacitance increases from zero to $0.6 \mu \mathrm{F}$. This is the so-called bypass effect of the capacitance.

Now, we consider the signal from a narrow square-wave driving voltage. This driving-voltage can be expressed, both in the time domain and in the frequency domain, by the Heaviside unit step functions:

$$
\begin{gathered}
u_{1}(t)=\left[H(t)-H\left(t-t_{1}\right)\right] U_{0}, \\
U_{1}(j \omega)=\frac{U_{0}\left(1-\mathrm{e}^{-j \omega t_{1}}\right)}{j \omega} .
\end{gathered}
$$

In eq. (36), $U_{0}$ is the amplitude of the driving voltage signal; $t_{1}$ is the window width of the driving voltage signal, and $H(t)$ is the Heaviside unit step function.

As an example, we chose the following set of parameters for the square-wave driving voltage: $U_{0}=400 \mathrm{~V}$, and $t_{1}=1.0$ or $t_{1}=11.0$ (in units $\pi / \omega_{1}$ ). The calculated results for the waveforms and amplitude spectra of the driving voltage are shown in Figure 6(a)-(d). When these two driving voltage signals are used to excite the source transducer with parameters

Table 2 Physical and geometrical parameters of transducer and acoustic impedance of the coupling medium

\begin{tabular}{ccccccccc}
\hline$\rho \times 10^{3}$ & $d_{31} \times 10^{-12}$ & $\varepsilon_{33}^{T} \times 10^{-9}$ & $s_{12}^{E} \times 10^{-12}$ & $s_{11}^{E} \times 10^{-12}$ & $Z_{m} \times 10^{6}$ & $l_{b}$ & $a$ & \\
\hline $7.6 \mathrm{~kg} / \mathrm{m}^{3}$ & $-60 \mathrm{~m} / \mathrm{V}$ & $3.7613 \mathrm{~F} / \mathrm{m}$ & $-3.2 \mathrm{~m}^{2} / \mathrm{N}$ & $10.7 \mathrm{~m}^{2} / \mathrm{N}$ & $1.2205 \mathrm{~kg} /\left(\mathrm{m}^{2} \mathrm{~s}\right)$ & $8 \mathrm{~cm}$ & $0.8 \mathrm{~cm}$ & $12 \mathrm{~cm}$ \\
\hline
\end{tabular}


Table 3 The physical parameters of the borehole fluid and the formation around the borehole

\begin{tabular}{lccc}
\hline Propagation medium & $\rho \times 10^{3}\left(\mathrm{~kg} / \mathrm{m}^{3}\right)$ & $v_{p}(\mathrm{~m} / \mathrm{s})$ & $v_{s}(\mathrm{~m} / \mathrm{s})$ \\
\hline Borehole fluid & 1.2 & 1540 & \\
Formation & 2.16 & 5943 & 3200 \\
\hline
\end{tabular}

$R_{1 m}=0.8 \pi r_{b}^{2} Z_{m}$ and $C_{2}=0$, the waveforms and amplitude spectra of the radiated acoustic pressure signals are calculated, as shown in Figure 6(e) and (f). It is shown that the appearance time for the radiated acoustic pressure signal is almost the same as that for the driving voltage signal, i.e. the transmission delay caused by the electric-acoustic conversion from the source transducer is small. This is largely because an ideal narrow square-wave signal was taken as the driving voltage signal. For the narrow square-wave driving voltage, Figure 6(a)-(f) also show that: (1) the wider the time window, the greater the electrical power that is concentrated in the low frequency ranges; (2) the radiated acoustic pressure signal has a phase advance compared to the driving voltage signal; and (3) the radiated acoustic signal consists of two wavelets with a phase difference of $180^{\circ}$. The first wavelet is caused by the rising edge of the driving voltage signal and the second is caused by the falling edge of the driving voltage signal. The two wavelets gradually
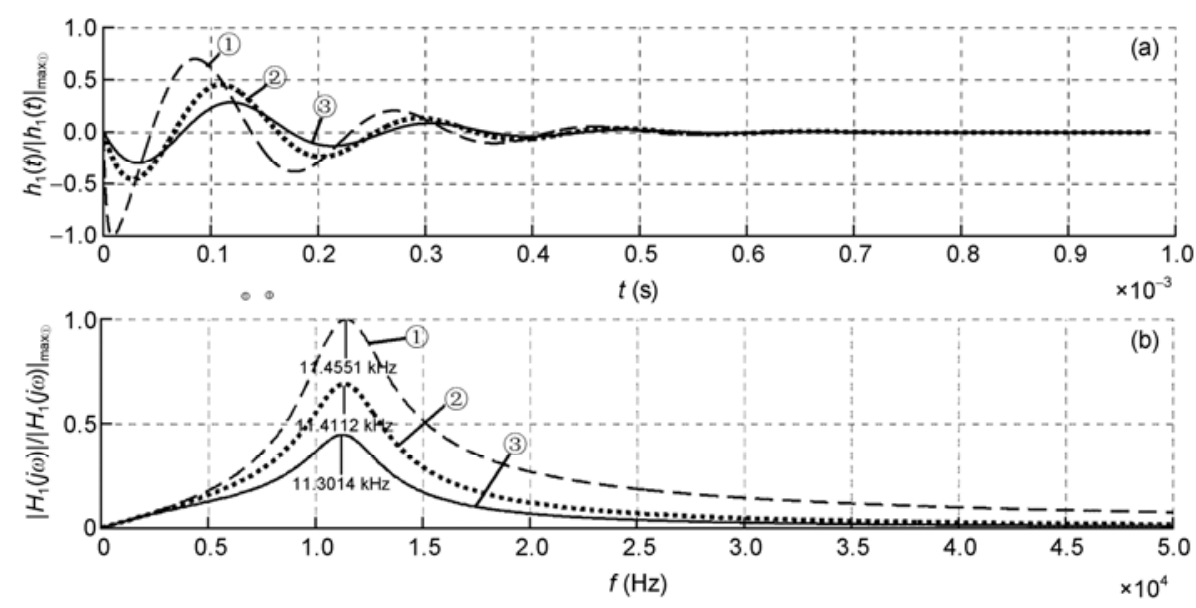

Figure 4 The normalized waveforms and amplitude spectra of $h_{1}(t)$ for both the different values of $R_{1 m}$ and the case where $C_{2}$ does not exist. The curves (1), (2) and (3) are the cases of $R_{1 m}$ with values of $\pi r_{b}^{2} Z_{m}, 5 \pi r_{b}^{2} Z_{m}$ and $12 \pi r_{b}^{2} Z_{m}$, respectively. (a) The waveforms normalized by $\left|h_{1}(t)\right|_{\max (1)}$ Here, $\left|h_{1}(t)\right|_{\max (1)}$ is the maximum of the absolute value of $h_{1}(t)$ with the parameter $R_{1 m}=\pi r_{b}^{2} Z_{m}$. (b) The amplitude spectrum normalized by $\left|H_{1}(j \omega)\right|_{\max (1)}$. Here, $\left|H_{1}(j \omega)\right|_{\max (1)}$ is the maximum of the absolute value of $H_{1}(j \omega)$ with the parameter $R_{1 m}=\pi r_{b}^{2} Z_{m}$.
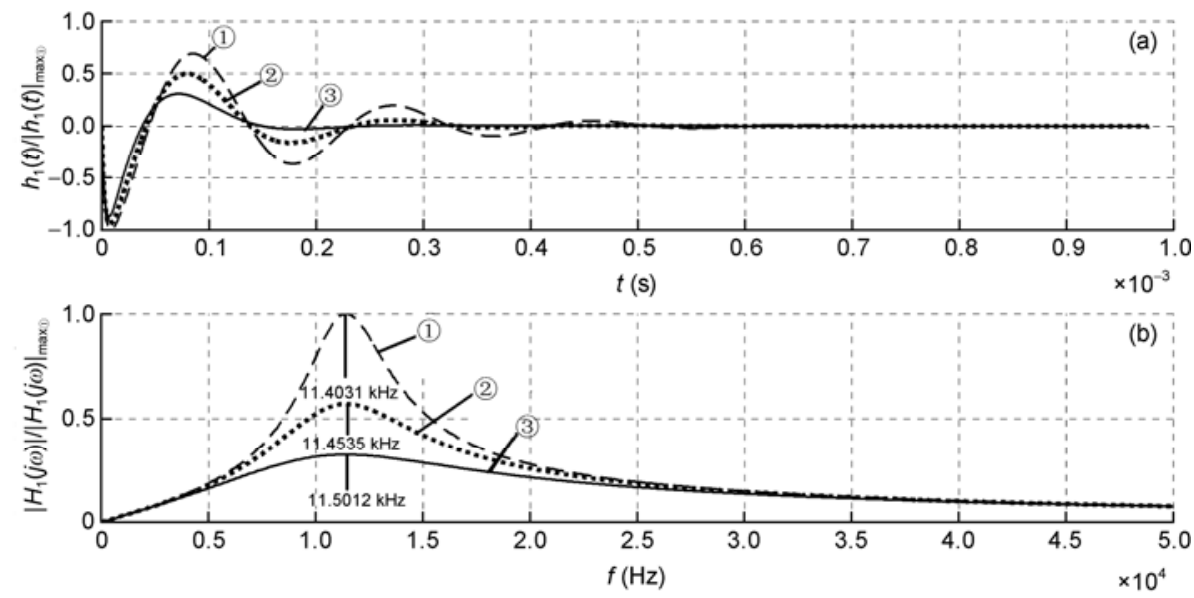

Figure 5 The normalized waveforms and amplitude spectra of $h_{1}(t)$ for both the different values of $C_{2}$ and the case of $R_{1 m}=0.8 \pi r_{b}^{2} Z_{m}$. The curves (1), (2) and (3) are the cases of $C_{2}=0, C_{2}=0.3 \mu \mathrm{F}$ and $C_{2}=0.6 \mu \mathrm{F}$, respectively. (a) The waveforms normalized by $\left|h_{1}(t)\right|_{\max (1)}$. Here, $\left|h_{1}(t)\right|_{\max (1)}$ is the maximum of the absolute value of $h_{1}(t)$ for the case where $C_{2}=0$. (b) The amplitude spectrum normalized by $\left|H_{1}(j \omega)\right|_{\max (1)}$. Here, $\left|H_{1}(j \omega)\right|_{\max (1)}$ is the maximum of the absolute value of $H_{1}(j \omega)$ for the case where $C_{2}=0$. 

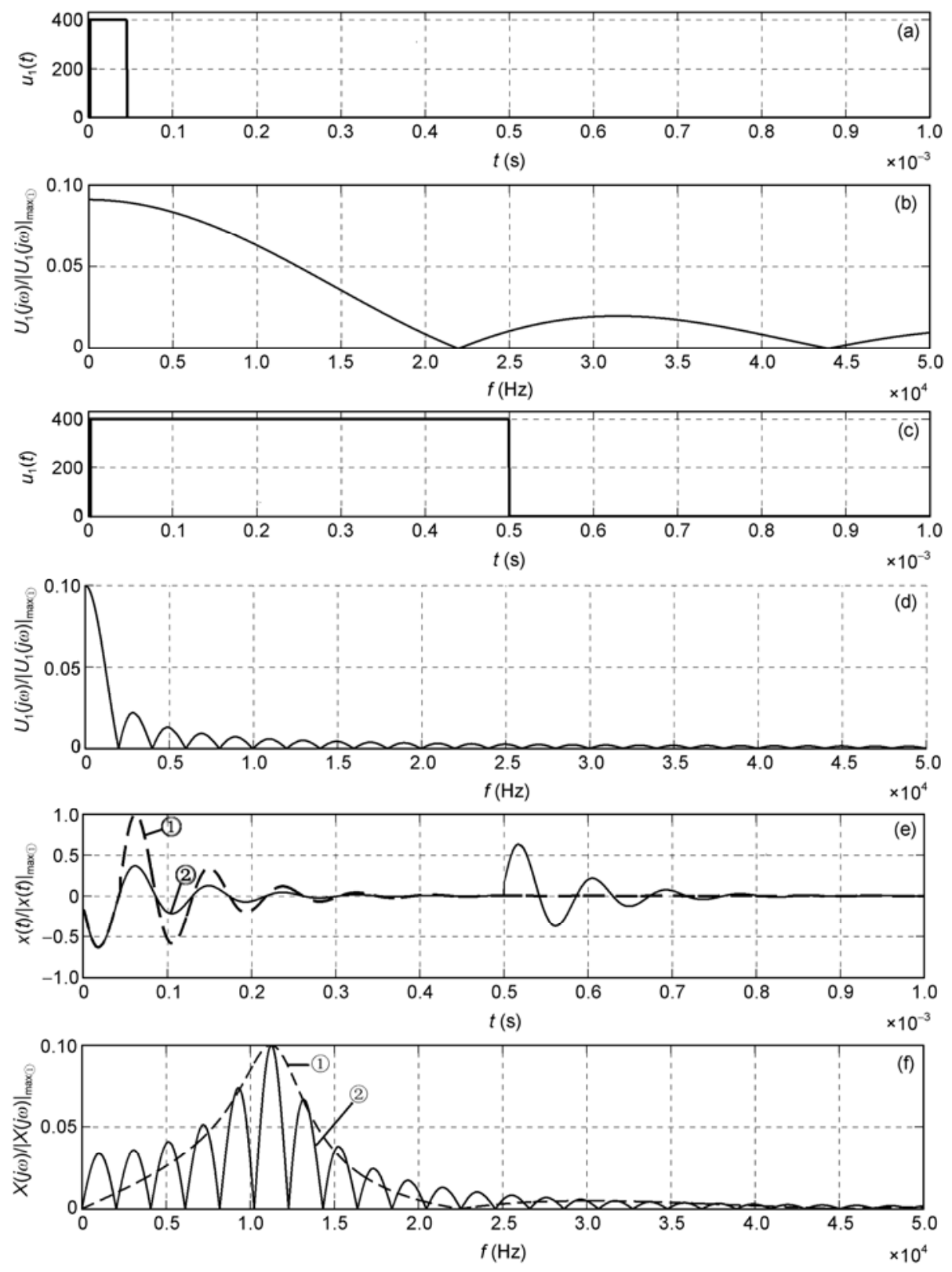

Figure 6 The normalized waveforms and amplitude spectra of $u_{1}(t)$, and those of $x(t)$. (a) and (c) The waveforms of $u_{1}(t)$. (b) and (d) The normalized amplitude spectra of $u_{1}(t)$. The amplitude spectra are normalized by $\left|U_{1}(j \omega)\right|_{\max (1)}$. Here, $\left|U_{1}(j \omega)\right|_{\max (1)}$ is the maximum of the absolute value of $U_{1}(j \omega)$ with a window width of $t_{1}=\pi / \omega_{1}$. (e) The normalized waveforms of $x(t)$. Curve (1) is the waveform of $x(t)$ for the case of a window width of $t_{1}=\pi / \omega_{1}$ and curve (2) is

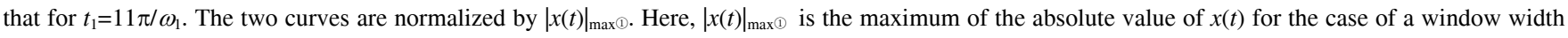
of $t_{1}=\pi / \omega_{1}$. (f) The normalized amplitude spectrum of $x(t)$. Curve (1) is the amplitude spectrum when $t_{1}$ is equal to $\pi / \omega$ and curve (2) is that when $t_{1}$ is equal to $11 \pi / \omega_{1}$. Two curves are normalized by $|X(j \omega)|_{\max (1)}$. Here, $|X(j \omega)|_{\max (1)}$ is the maximum of the absolute value of $X(j \omega)$ when $t_{1}$ is equal to $\pi / \omega_{1}$.

separate with increasing window width. When the time window is equal to $\pi / \omega_{1}$, the two wavelets are coherent, and the amplitude of the radiated acoustic pressure signal is at its highest.

\subsection{Effect of the receiver transducer and media on the acoustic logging signal}

(i) Acoustic-electric impulse response and corresponding amplitude spectrum for receiver transducer. Figure 7 shows the calculated results for the waveforms and amplitude spectra of the acoustic-electric impulse responses of the receiver transducer when the load resistance $\left(R_{3}\right)$ is equal to $500 \Omega$ and $50 \mathrm{k} \Omega$. The corresponding center frequencies are at $12.532 \mathrm{kHz}$ and $13.201 \mathrm{kHz}$, respectively. The maxima of the acoustic-electric impulse response, and that of the corresponding amplitude spectrum and the center frequency $f_{3}$, increase with respect to $R_{3}$. The absolute values 

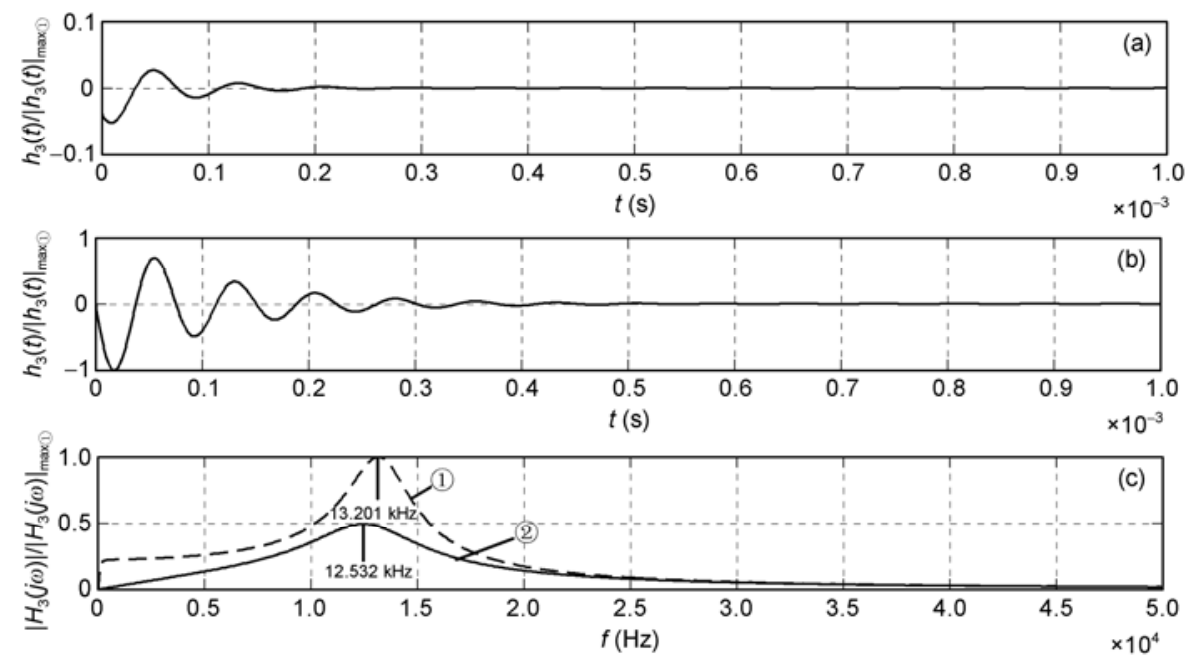

Figure 7 The normalized waveforms and amplitude spectra of $h_{3}(t)$. (a) The normalized waveform of $h_{3}(t)$ with output impedance of $R_{3}=500 \Omega$. This waveform is normalized by $\left|h_{3}(t)\right|_{\max (1)}$. Here, $\left|h_{3}(t)\right|_{\max (1)}$ is the maximum of the absolute value of $h_{3}(t)$ with the output impedance of $R_{3}=50 \mathrm{k} \Omega$. (b) The normalized waveform of $h_{3}(t)$ for the parameter $R_{3}=50 \mathrm{k} \Omega$. (c) The normalized amplitude spectra corresponding to $h_{3}(t)$. Curves (1) and (2) are the amplitude spectra corresponding to $h_{3}(t)$ when $R_{3}$ is equal to $50 \mathrm{k} \Omega$ and $500 \Omega$, respectively and both of them are normalized by $\left|H_{3}(j \omega)\right|_{\max (1)}$. Here, $\left|H_{3}(j \omega)\right|_{\max (1)}$ is the maximum of the absolute value of $H_{3}(j \omega)$ with the parameter $R_{3}=50 \mathrm{k} \Omega$.

of the phase shift also vary with respect to the different values of $R_{3}$.

(ii) Effect of the media and acoustic-electric conversion of the receiver transducer. Now we consider a squarewave signal with a window width of $\pi / \omega_{1}$ that acts as the driving-voltage signal of the source transducer.

Based on eq. (35), we have calculated the waveform and amplitude spectrum of the normalized acoustic logging signal at the position of the receiver transducer, as shown in Figure 8(a) and (b). At this position, the acoustic logging signal has a head wave arrival time $\left(t_{p}\right)$ of $373.8971 \mu \mathrm{s}$, with a normalized head wave amplitude of -0.5835 . When the receiver transducer is loaded with impedances of $500 \Omega$ and $50 \mathrm{k} \Omega$, the waveforms and amplitude spectra of the output electric signals from the receiver transducer are calculated and are shown in Figure 8(c)-(f). The appearance times of the head waves are given as $375.5621 \mu$ s and $374.5985 \mu \mathrm{s}$. The transmission delays of the acoustic-electric conversion from the receiver transducer are $1.6665 \mu \mathrm{s}$ and $0.7014 \mu \mathrm{s}$. The calculated results in Figure 8 also show that the acoustic-electric conversion of the receiver transducer can cause variations in the amplitude, phase and the amplitude spectrum. When the acoustic logging signal shown in Figure 8(a) is injected into the receiver with a load impedance of $500 \Omega$, the head wave amplitude of $u_{3}(t)$ is positive and equal to 0.1636 , as shown in Figure 8(c); when this signal is injected into the receiver with a load impedance of $50 \mathrm{k} \Omega$, the value of $u_{3}(t)$ is negative and equal to -0.3435 , as shown in Figure 8(e). These two head wave amplitudes have phase distances of $180^{\circ}$, and the relative error is calculated as

$$
\frac{|-0.3435|-|0.1636|}{|-0.3435|} \times 100 \%=52.37 \% \text {. }
$$

(iii) Interrelationship between acoustic logging signal property and acoustic-electric conversion. To calculate the interrelationship between the acoustic logging signal properties and the acoustic-electric conversion of the receiver transducer, we use the Tsang wavelet [6] in place of the acoustic pressure signal $x(t)$ radiated by the source transducer. The Tsang wavelet is expressed as

$$
x(t)=4 \alpha t \mathrm{e}^{-\alpha t} \sin \left(\omega_{0} t\right) H(t),
$$

where $\alpha$ is the damping coefficient and $\omega_{0}$ is the center frequency of the wavelet.

In the following calculations, we choose the parameters $\omega_{0}=2 \pi \times 10^{4} \mathrm{rad} / \mathrm{s}$, and $R_{3}=50 \mathrm{k} \Omega$.

The waveform and amplitude spectrum of the normalized Tsang wavelet with parameter $\alpha=0.3 \omega_{0} / \pi$ are calculated as shown in Figure 9(a) and (b). The waveform and amplitude spectrum of the Tsang wavelet propagating from the source transducer position to the receiver transducer position are shown in Figure 9(c) and (d). It is found that the head wave arrival time for the acoustic pressure signal is $373.8971 \mu \mathrm{s}$; the normalized head-wave amplitude is -0.2959 ; and the center frequency is $10.2032 \mathrm{kHz}$. The waveform and amplitude spectrum of the electric signal output from the receiver transducer are calculated as shown in Figure 9(e) and (f). In this case, the head wave appearance time of the electric signal is $376.4983 \mu \mathrm{s}$; the normalized head wave amplitude is 0.1078 ; and the center frequency is $10.4123 \mathrm{kHz}$. The created acoustic-electric transmission delay from the receiver transducer is $2.6012 \mu \mathrm{s}$.

Using the parameter $\alpha=0.6 \omega_{0} / \pi$, the waveform and amplitude spectrum of the normalized Tsang wavelet are shown in Figure 10(a) and (b); and those of the Tsang wavelet 

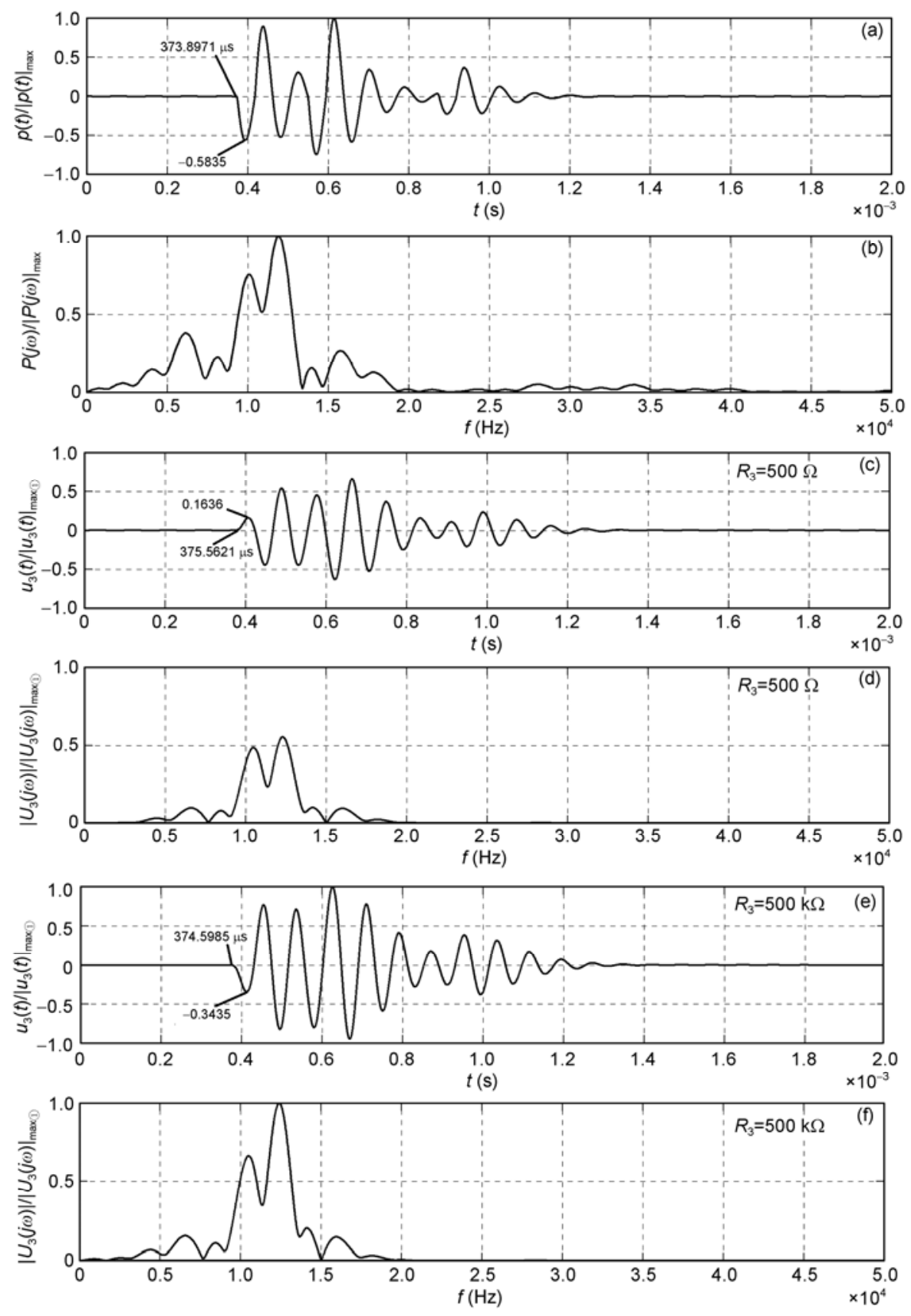

Figure 8 The normalized waveforms and amplitude spectra of $p(t)$ and those of $u_{3}(t)$. (a) The normalized waveform of $p(t)$. (b) The normalized amplitude spectrum corresponding to $p(t)$. (c) The normalized waveform of $u_{3}(t)$ for the parameter $R_{3}=500 \Omega$. This waveform is normalized by $\left|u_{3}(t)\right|_{\max (1)}$. Here, $\left|u_{3}(t)\right|_{\max (1)}$ is the maximum of the absolute value of $u_{3}(t)$ for the parameter $R_{3}=50 \mathrm{k} \Omega$. (d) The normalized amplitude spectrum corresponding to $u_{3}(t)$ for the parameter

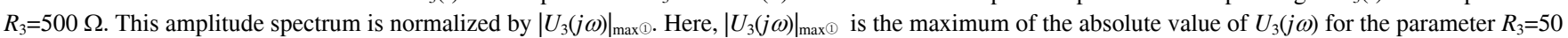
$\mathrm{k} \Omega$. (e) The normalized waveform of $u_{3}(t)$ for the parameter $R_{3}=50 \mathrm{k} \Omega$. (f) The normalized amplitude spectrum corresponding to $u_{3}(t)$ for the parameter $R_{3}=50 \mathrm{k} \Omega$.

propagating from the source transducer position to the receiver transducer position are shown in Figure 10(c) and (d). These figures show that the head wave arrival time of the acoustic pressure signal is $373.8971 \mu \mathrm{s}$; the normalized head wave amplitude is -0.4347 ; and the center frequency is $10.5769 \mathrm{kHz}$. As shown in Figure 10(e), the electric signal output time of the receiver transducer is $377.0857 \mu \mathrm{s}$ and the normalized head wave amplitude is 0.1596 . Figure 10(f) shows that the center frequency is at $10.6538 \mathrm{kHz}$. The created acoustic-electric transmission delay is $3.1886 \mu$ s.

The relative constrast between the head amplitudes of the above two normalized Tsang wavelets, propagating from the source to the receiver, is $\frac{|-0.4947|-|-0.2959|}{|-0.4947|} \times 100 \%$ 

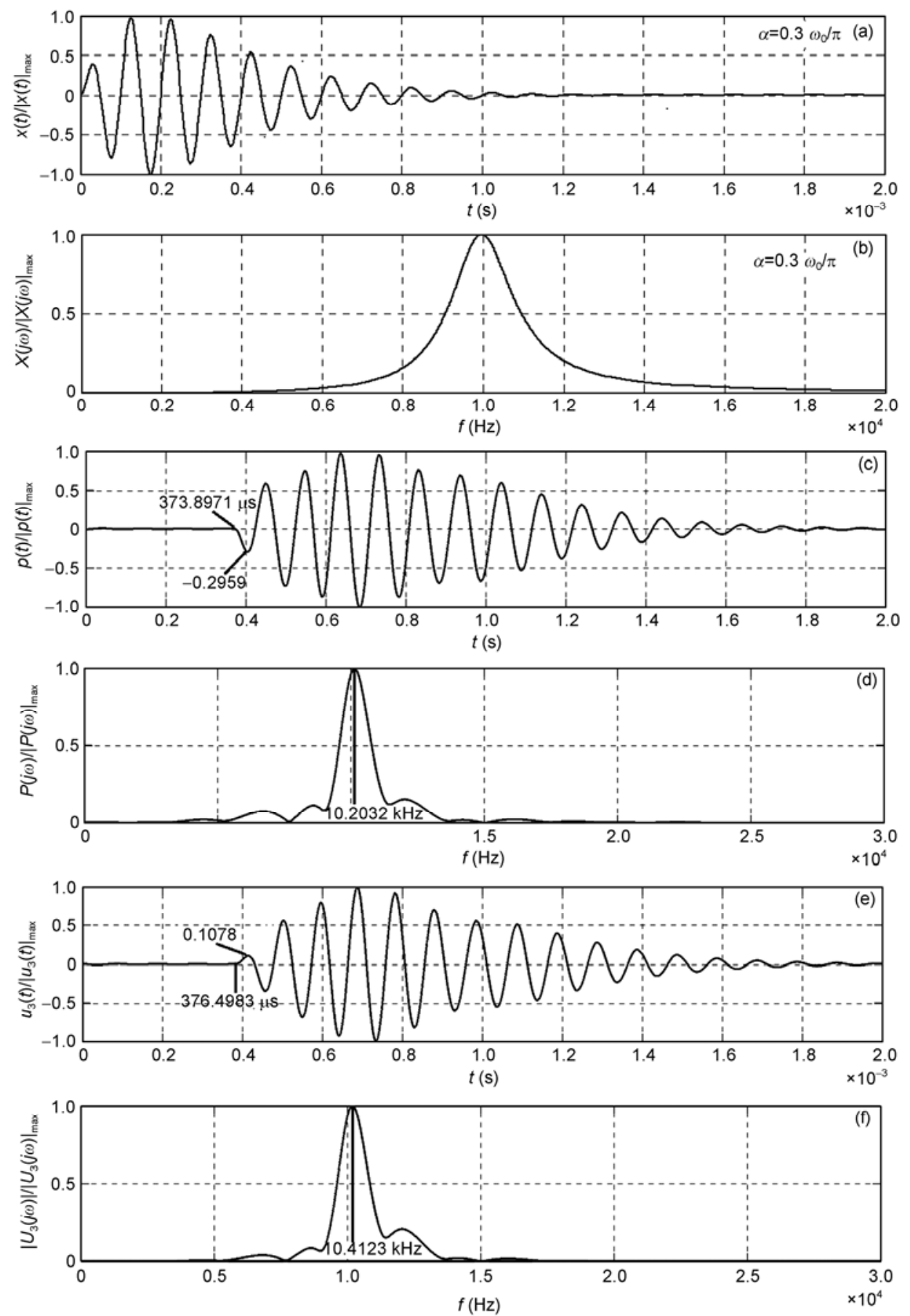

Figure 9 The effects of the properties of $p(t)$ on the amplitude variation of $u_{3}(t)$ and transmission delay for a Tsang wavelet with parameters of $\omega_{0}=2 \pi \times 10^{4}$ $\mathrm{rad} / \mathrm{s}$ and $\alpha=0.3 \omega_{0} / \pi \mathrm{s}^{-1}$. (a) and (b) The normalized waveform and amplitude spectrum of the Tsang wavelet. (c) and (d) The normalized waveform and amplitude spectrum of $p(t)$. (e) and (f) The normalized waveform and amplitude spectrum of $u_{3}(t)$.

$=40.09 \%$, while that between the corresponding output electric signals from the receiver is (0.1596-0.1078)/0.1078 $\times 100 \%=32.46 \%$. Therefore, the effect of the acousticelectric conversion of the receiver on the head wave amplitude of the acoustic logging signal is fairly large. The difference between the two transmission delays above is $0.5874 \mu \mathrm{s}$ (=3.1886 $\mu \mathrm{s}-2.6012 \mu \mathrm{s})$.
For the acoustic velocity of the acoustic logging signal propagating in a rock formation around the borehole with a source-receiver distance of $1.3224 \mathrm{~m}$, the relative errors caused by the acoustic-electric conversion are calculated to be only $1.23 \%$ and $1.51 \%$ for the above two cases. For a source-receiver distance of $0.8 \mathrm{~m}$ and a transmission delay of $3.1886 \mu \mathrm{s}$, the calculated relative error for the above 

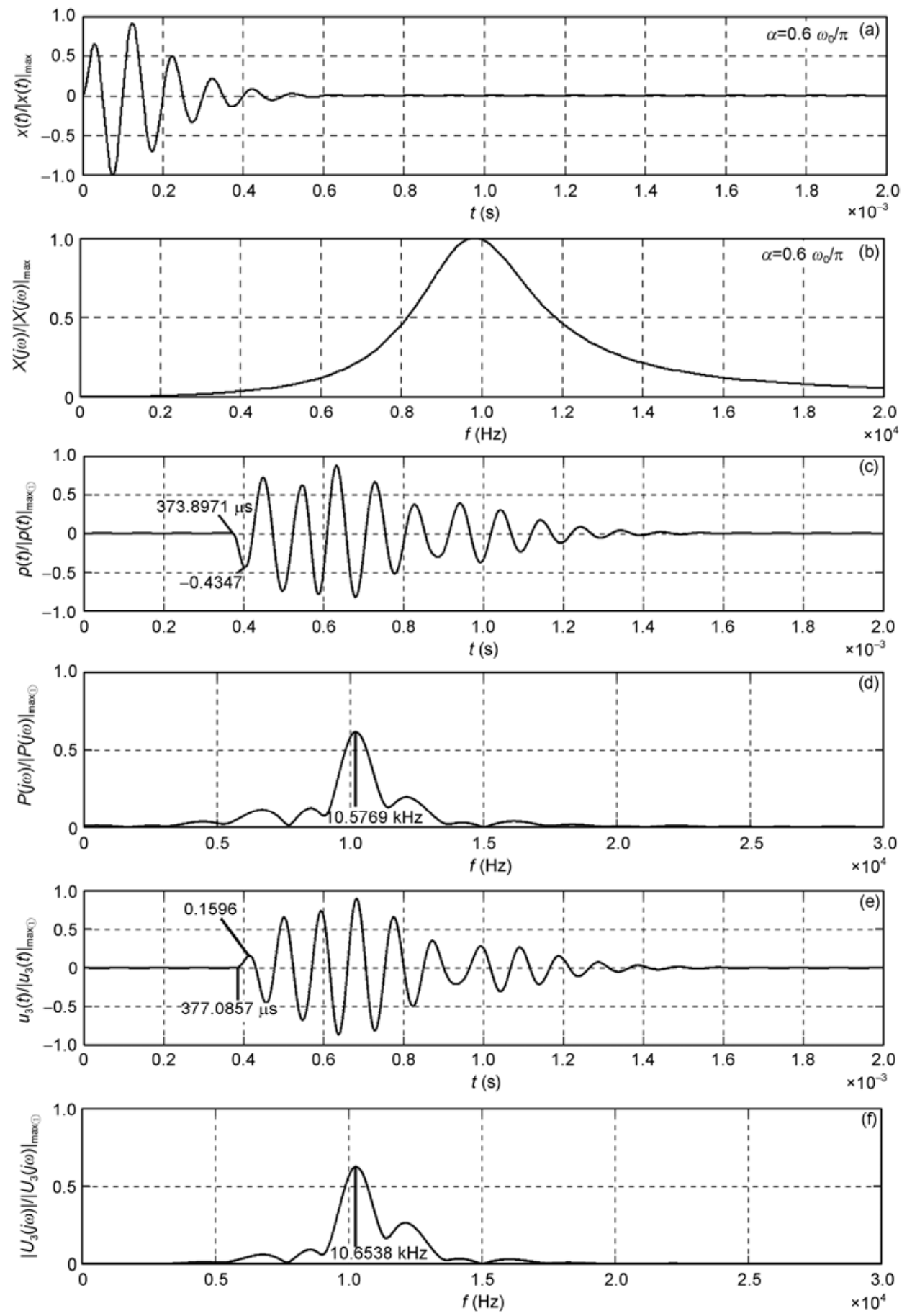

Figure 10 The effect of the properties of $p(t)$ on the amplitude variation of $u_{3}(t)$ and transmission delay for a Tsang wavelet with parameters of $\alpha=0.6 \omega_{0} / \pi \mathrm{s}^{-1}$ and $\omega_{0}=2 \pi \times 10^{4} \mathrm{rad} / \mathrm{s}$. (a) and (b) The normalized waveform and amplitude spectrum of the Tsang wavelet. (c) and (d) The waveform of the amplitude spectrum of $p(t)$. Both of them are normalized by $\left|p(t)_{\max 11}\right|$ and $\mid P(j \omega)_{\max (1)}$, respectively, where $\left|p(t)_{\max 11}\right|$ is the maximum of the absolute value of $p(t)$ and $\left|P(j \omega)_{\max 11}\right|$ is that of the absolute value of $P(j \omega)$ for the parameter $\alpha=0.3 \omega_{0} / \pi \mathrm{s}^{-1}$. (e) and (f) The waveform of the amplitude spectrum of $u_{3}(t)$. Both of them are normalized by $\left|u_{3}(t)_{\max 11}\right|$ and $\left|U_{3}(j \omega)_{\max 11}\right|$, respectively, where $\left|u_{3}(t)_{\max (1)}\right|$ is the maximum of the absolute value of $u_{3}(t)$, and $\left|U(j \omega)_{\max (1)}\right|$ is that of the absolute value of $U(j \omega)$ for the parameter $\alpha=0.3 \omega_{0} / \pi \mathrm{s}^{-1}$.

acoustic velocity is $2.58 \%$. This indicates that the shorter the source-receiver distance is, the greater the relative error becomes.

The calculated results above show that in most of the studied cases, because of the electric-acoustic conversions, the transducer can cause amplitude variation in an acoustic signal and transmission delay. However, the appropriate choice of the driving voltage signal, such as an ideal narrow 
square-wave driving voltage or a gated sinusoid driving voltage with the center frequency of the source transducer, may decrease the electric-acoustic transmission delay significantly [13]. The acoustic-electric transmission delay created is relative not only to the acoustic-electric conversion properties of the receiver transducer, but also to the wave properties at the receiver transducer position.

Now, we extend the results shown in Figures 8-10 to discussions of the first interface cement bond quality of a cased well, where the first interface refers to the interface between the casings and the cement layer. For the cement bond logging of the cased well, the cement bond quality of the first interface is evaluated from the head wave amplitude of an acoustic logging signal. If the received head wave amplitude is large, it indicates that the cement bond quality is poor; otherwise, the cement bond quality is good.

We must now look at the significance of the effects of transducers in practical engineering work. We consider a practical case in the petroleum logging engineering industry. Assume that a cased well has a very poor cement bond quality at the first interface, and then the absolute value of the head wave amplitude of an acoustic logging signal arriving at the position of receiver transducer should be large. During the cement bond logging of the cased well, the acoustic-electric conversion of the receiver transducer may cause a large variation in the head wave amplitude of the acoustic logging signal. This obviously leads to a serious problem in engineering practice. By ignoring the effect of the acoustic-electric conversion from the receiver transducer on acoustic logging signals, a logging engineer may misinterpret a very poor cement bond quality as good or a good cement bond quality as poor for the first interface of the cased well. For a long source-receiver distance used to measure the propagation speed of an acoustic signal in rock, the effect of the transmission delay of several microseconds caused by the acoustic-electric conversion on the measurement accuracy is small. However, for a short source-receiver distance, especially for a small-sized rock sample, the effect of this transmission delay on the accuracy of the measured propagation speed cannot be neglected, even though the acoustic signal wavelet has a higher center frequency.

Therefore, during the measurement of the parameters of acoustic signal wavelets in rock, including cement bond quality logging, the acoustic velocity logging, the measurement of the propagation speed of the acoustic signal wavelet in the rock samples, the amplitude variation and the transmission delay caused by the acoustic-electric conversion of the receiver transducer must all be considered.

\section{Conclusions}

We have studied the effects of the electric-acoustic and acoustic-electric conversions of the transducers on the amplitude, transmission delay, amplitude spectrum, and phase of the acoustic logging signal. From the analyses and discussions in the earlier Sections, we have reached the following conclusions.

(i) The proper choice of the driving voltage signal can significantly improve the electric-acoustic conversion efficiency of the source transducer. For example, a narrow square-wave driving voltage with a time window of $\pi / \omega_{1}$ produces the largest amplitude for the radiated acoustic signal.

(ii) The acoustic-electric conversion of the receiver transducer can cause serious variations in the amplitude, phase, waveform, and amplitude spectrum of the acoustic logging signal. It can also create an acoustic-electric transmission delay.

(iii) The injected acoustic signal on the receiver transducer is different. The amplitude variation and transmission delay of the acoustic logging signal caused by the acoustic-electric conversion is also different. Using two receivers at different ranges to measure the arrival time difference of the acoustic logging signal can partly decrease the transmission delay of the acoustic logging signal.

(iv) The practical propagation time of an acoustic logging signal in the medium is equal to the total transmission time of the signal wavelet in the acoustic logging transmission network minus the sum of the electric-acoustic and acoustic-electric transmission delays caused by the transducers, where the medium is the borehole fluid and the formation around the borehole. The transmission delays should not be neglected for accurate measurement of the propagation time of the acoustic signal, especially for the case of a short source-receiver distance. By appropriate choice of the driving voltage signal, the electric-acoustic transmission delay can be decreased significantly, although it cannot be completely eliminated.

It is worth noting that the analysis described in this work is useful for accurate measurements of both the amplitude and the propagation velocity of the acoustic logging signal for both acoustic velocity logging and cement bond logging.

This work was supported by the National Natural Science Foundation of China (40974078).

1 Wyllie M R J, Gregory R E, Gardner L W. Elastics wave velocities in heterogeneous and porous media. Geophysics, 1956, 21: 41-70

2 Cong J S, Wang X M, Xu D L, et al. Experimental studies on the effects of porosity on acoustic resonance spectroscopy for synthetic porous rock samples in a cylindrical resonant cavity. Chin Sci Bull, 2008, 53: 978-983

3 Fa L, Castagna J P, Dong H F. An accurately fast algorithm of calculating reflection/transmission coefficients. Sci China Ser G: Phys Mech Astron, 2008, 51: 823-846

4 Fa L, Castagna J P, Zeng Z W, et al. Effects of anisotropy on time-depth relation in transversely isotropic medium with a vertical axis of symmetry. Chin Sci Bull, 2010, 55: 2243-2251

5 Aki K, Rechards P G. Quantitative Seismology: Theory and Methods. San Francisco: W. H. Freeman, 1980

6 Tsang L, Rader D. Numerical evaluation of the transient acoustic 
waveform due to a point source in a fluid-filled bore hole. Geophysics, 1979, 44: 1706-1720

7 Gibson Jr R L, Peng C. Low- and high-frequency radiation from seismic sources in cased boreholes. Geophysics, 1994, 59: 1780-1785

8 Cheng $\mathrm{C} \mathrm{H}$, Toksöz M N. Elastic wave propagation in a fluid-filled borehole and synthetic acoustic logs. Geophysics, 1981, 46: 10421053

9 Piquette J C. Method for transducer suppression. I: Theory. J Acoust Soc Am, 1992, 92: 1203-1213

10 Piquette J C. Method for transducer suppression. II: Experiment. J
Acoust Soc Am, 1992, 92: 1214-1221

11 Piquette J C, Forsythe S E. Transducer transient suppression: Generalized methods of analysis. J Acoust Soc Am, 1996, 100: 1577-1583

12 Fa L, Castagna J P, Hovem J M. Derivation and simulation of source function for acoustic logging. IEEE Int Ultrason Symp Proc, 1999, 1: $707-710$

13 Fa L, Castagna J P, Hovem J M, et al. An acoustic-logging transmission-network model. J Acoust Soc Am, 2002, 111: 2158-2165

14 Auld B A. Acoustic Fields and Waves in Solids. New York: WileyIntersciences, 1973

Open Access This article is distributed under the terms of the Creative Commons Attribution License which permits any use, distribution, and reproduction in any medium, provided the original author(s) and source are credited. 